\title{
Early prediction of Alzheimer's disease by examining changes in eye parameters
}

\begin{abstract}
Alzheimer's disease (AD) is a type of senile dementia which can be called as a brain and eye disorder that affects memory and the living condition of the people. AD is presently becoming an issue of therapeutic, social and community health concern. There are lot of tests and imaging modalities to be performed for an effective diagnosis of the disease. Conventional clinical decision making systems are more manual in nature and ultimate conclusion in terms of exact diagnosis is remote. In this case, the use of advanced biomedical engineering technology will definitely helpful for making diagnosis. There are several imaging techniques used in medical practice for the prediction of AD. The most popular of them are Magnetic Resonance Imaging, Positron Emission Tomography and Single Photon Emission CT Scanning. From the several investigations made on Alzheimer's disease it is clearly identified that there are some parameter changes on the retina of the eye of the $\mathrm{AD}$ patients. Therefore retina can be used as a biomarker for the early diagnosis of AD. In this review article we have focus on the changes in the parameter of retina by examining eye with the help of fundus imaging and Optical Coherence Tomography (OCT). Therefore the prediction of $\mathrm{AD}$ can be done using the above tests with the combination of soft computing techniques.
\end{abstract}

Keywords: Alzheimer's disease, retina, early diagnosis, fundus imaging, OCT
Volume 5 Issue 6 - 2017

\section{Sandeep CS, Sukesh Kumar A}

Department of ECE, College of Engineering, University of Kerala, Trivandrum, India

Correspondence: Sandeep CS, Department of ECE, College of Engineering, University of Kerala, Trivandrum, India, Email sandeepcs07nta@gmail.com

Received: June 08, 2017 | Published: August 22, 2017
Abbreviations: AD, alzheimer's disease; OCT, optical coherence tomography; CT, computed tomography; MRI, magnetic resonance imaging; PET, positron emission tomography; SPECT, single-photon emission computed tomography; CSF, cerebrospinal fluid; ONH, optical nerve head; RNFL, retinal nerve fibre layer

\section{Introduction}

$\mathrm{AD}$ is an irreversible progressive neurodegenerative disorder which means once it is affected, cannot be cured. ${ }^{1,2}$ It is a memory and behavioral disturbance which leads to intense and eternal loss of cognitive impairment. ${ }^{3}$ The Patients with $\mathrm{AD}$ starts initially having problems with remembering routine functions, after that it continues to have incapability to identify the distant relatives and different jobs that have been done earlier. ${ }^{4}$ After some years, AD patients seem to have complete memory impairment and full loss of control. ${ }^{5}$ The two most important causes of AD are plaques and tangles that accumulate on the neurons which blocks the signals from brain to neuron and viceversa. ${ }^{6}$ The entorhinal cortex and hippocampal regions are the part of the brain where the disease seems to be appear earlier, but increasing evidence shows that the internal layers of the retina, is also affected. ${ }^{7}$ There are different types of tests for diagnosing AD. Some of them are illustrated below. Neuropsychological test helps the AD subject for evaluating its initial condition and cognitive screening. ${ }^{8,9}$ The economic imaging modalities which are used for diagnosing $\mathrm{AD}$ areComputed tomography (CT) scan or magnetic resonance imaging (MRI). These modalities will help to figure out irregularities in the brain structure and brain tissues which are the reason for memory impairment. In addition to this, the clinicians want to rule out whether the abnormalities in the brain is due to normal ageing or other previous accidents or diseases in connection with brain such as tumor, hydrocephalus, history of strokes, and white matter disease. The MRI is more sensitive than CT to find out irregularities in white matter. ${ }^{10}$ Apart from CT and MRI other neuroimaging modalities used in practice are Positron Emission Tomography (PET), Single-Photon
Emission Computed Tomography (SPECT), and functional MRI, are not using as a part of regular dementia diagnosis. But in current medical practice, functional imaging may be helpful in the diagnosis of dementia of Alzheimer's type. ${ }^{11-13}$ Cerebrospinal fluid (CSF) examination which was done earlier is now not a part of the routine examination of $\mathrm{AD},{ }^{14}$ the $\mathrm{CSF}$ range of tau and amyloid beta protein helps to diagnose $\mathrm{AD}$. We know that the dementia of Alzheimer's type is a brain related disease, but recent studies and reports shows that there is evidence of visual problems associated in the early stages of AD. These includes problems in reading, unable to find objects, incapable of seeing deep objects, difficulty to detect moving objects, difficulty in recognizing color features. ${ }^{15}$ The studies that were done previously shown that this happens due to the dysfunction in the visual cortex. But the presently made reports show that there are proofs available that the anterior visual pathways are also connected with AD. These include degeneration of nerves in optics and retinal ganglion cells loss. ${ }^{16,17}$ The main reason in the visual disabilities is due to the deposits of amyloid beta $(\mathrm{A} \beta)$ protein and acetylcholine has been found in the retina. The property by which $A \beta$ causes the retinal neurons death is not understood totally, but same type of neuronal dysfunction happen in the retina and brain of $\mathrm{AD}$ patients have been demonstrated. ${ }^{16,18}$ The starting of AD pathology may occur in the visual association area. ${ }^{19}$ $\mathrm{AD}$ shows loss of visual properties early in the disease and functional losses correlate with cognitive losses. The losses of visual function in $\mathrm{AD}$ have different aspects that are usual with neuronal losses affecting the eye, such as age-related macular degeneration and glaucoma. ${ }^{20}$ In normal cases without memory impairment there was an absence of such pathology in the hippocampus area where the symptoms of $\mathrm{AD}$ begins, which processes memory. The loss of visual function other than acuteness may be the initial significant indication of AD. Agerelated macular degeneration degrades all frequencies of contrast sensitivity; demonstrate color disorders across all wavelengths, and decrease in foveal detection of motion. ${ }^{21-23}$ The researchers are stating that instead of starting in the brain's regions that process memory, 
such as the hippocampus, $\mathrm{AD}$ may actually start in the portion of the brain that integrates visual function or the vision associated area. In people with $\mathrm{AD}$, contrast sensitivity degrades in the lower spatial frequencies are found motion perception, ${ }^{20}$ i.e., the ability to detect movement, is reduced, ${ }^{24-26}$ there are visual field defects ${ }^{27,28}$ and color discrimination of blue, short wave length hues have been found to be reduced. ${ }^{29}$ They found that in the visual association area of more than $50 \%$ of those who were considered cognitively normal there were no memory impairments, but there were findings of beta amyloid and neurofibrillary tangles, the presence of which are associated with Alzheimer's disease. All of these tests and scans can help to show the memory recall of a patient and the possible areas where the patient lacks deficiency. Using these tests can be helpful to find the types of treatment plans that can be given, however neuropsychological tests alone are not helpful in detecting early AD. Trials were then conducted combining neuropsychological tests with clinical tests and various imaging technologies. For an effective and early diagnosis of $\mathrm{AD}$, a population based study is necessary and required, which gives an idea about the various tests involved in determining AD. A biomarker can be called as an indicator to measure the severity or presence of some disease state. More generally a biomarker is anything that can be used as an indicator of a particular disease state or some other physiological state of an organism. The retina of the eye can be considered as a biomarker as there are changes in the layer of the retina of AD Patients. In this paper we have focus on fundus imaging and Optical Coherence Tomography. The author's previous works in the area of Biomedical Engineering will definitely help to develop a new tool using latest biomedical methods for the solution of the early diagnosis of AD.

\section{Retinal images and AD}

The images taken on the eye are used in the analysis of retinal ganglion function of the cell and loss of cell in the retinal layer. The pictures of retina images taken with different photographic instruments are used to find the cell's retinal nerve fiber layer degradation, a method that indirectly examines the loss of cell function. The nerve fiber layer is made up of several individual cell called as axons. Measuring the integrity of the cell fiber layer assesses loss in cell function is a big task. The pictures from photographic machines helps to store optic nerve parameters involved in cell loss such as tilt, depth and vasculature. All the studies related photographic images of the eye have shown a change in the retinal nerve fiber layer loss in patients with $\mathrm{AD}$ compared to control subjects. ${ }^{20}$ Also the studies which include only early-onset AD concluded that the optic nerve is not at all degraded and hence there is no change in the retinal ganglion cells.

\section{Fundus imaging}

Fundus camera records the neurosensory tissue in our eyes which translates the optical images into the electrical impulses. The retina of the eye can be captured directly as the pupil is used as incoming and outgoing path for the fundus camera's illuminating and imaging light rays. The patient sits at the fundus camera with their chin in a chin rest and their forehead against the bar. An ophthalmologist focuses and aligns the fundus camera. A flash light emits from the camera as the ophthalmologist presses the shutter release, creating a fundus photograph. Fundus imaging can be performed with the help of filters with different colors, or with specialized dyes. A fundus camera is a specialized low power microscope with an attached camera. Its design is based on the indirect ophthalmoscope. Fundus cameras are described by the angle of view - the optical angle of acceptance of the lens. An angle of $30^{\circ}$, considered the normal angle of view, creates a film image 2.5 times larger than life. Fundus cameras with wide angle capture eye images between $45^{\circ}$ and $140^{\circ}$ and provide the magnification of the retina lower than the previously discussed. A narrow angle fundus camera has an angle of view of $20^{\circ}$ or less. A standard approach in automatic fundus image analysis includes image acquisition, preprocessing, image segmentation, feature extraction, feature selection and classification. Image acquisition is done by fundus camera which is shown in Figure 1.

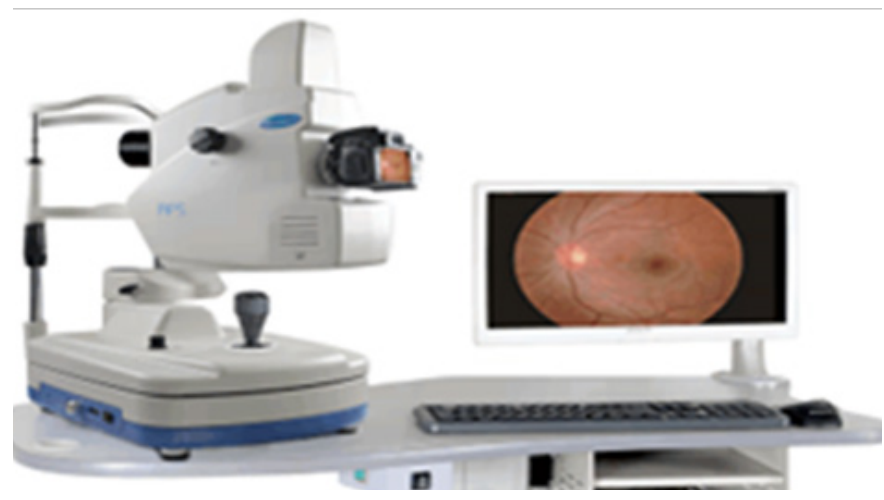

Figure I Fundus camera and fundus image on the screen.

\section{Optical coherence tomography}

Optical Coherence Tomography (OCT) is a promising as well as noninvasive retina imaging technique that provides cross-sectional images of the eye retina with quality resolution pictures. During the OCT process, six linear scans centered on the Optical Nerve Head $(\mathrm{ONH})$ is obtained, and the OCT software derives the $\mathrm{ONH}$ parameters in an automatic manner. The scan also gives horizontal and vertical cup-to-disc ratios. For the measurement of Retinal Nerve Fibre Layer (RNFL), the usable RNFL thickness circle scan mode which consists of 3 scans that are circular with twice the radius of $3.4 \mathrm{~mm}$ centered on the ONH should be used. Thus the overall, average and quadrant RNFL thicknesses should be calculated automatically. At the OCT scanning, the subject the clinicians should give instructions to fixed on a target internally to bring the $\mathrm{ONH}$ within view of the practioner. For the analysis of RNFL thickness the best-quality scan such as focused picture of the fundus, sufficient signal-to-noise ratio, and the presence of a centered, circular ring around the $\mathrm{ONH}$ should be chosen. The average of the RNFL thicknesses in each quadrant of the study eye should be compared with the AD patients and the normal subjects. The OCT device and OCT image is shown on Figure 2.

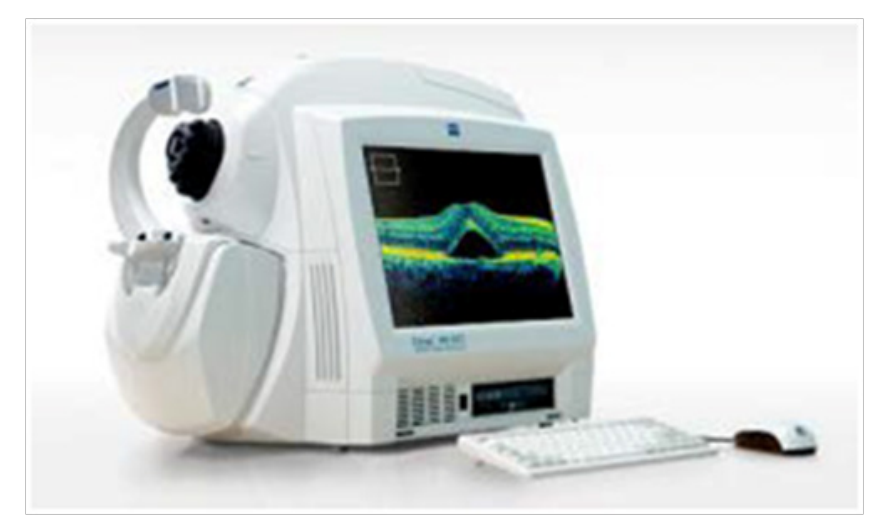

Figure 2 OCT device and OCT image on the screen. 


\section{Discussion}

Treatments used for $\mathrm{AD}$ also have implications for impact on the visual system. Cataracts currently are considered a biomarker for $\mathrm{AD}$, and this warrants further investigation. Glaucoma and $\mathrm{AD}$ are both neurodegenerative diseases, and neuroprotective treatments such as memantine are believed to be applicable to both diseases. Immunotherapy for amyloid beta has a positive impact on age-related macular degenerative disease in animal models. Therefore fundus imaging and OCT give every indication that the technologies can be practical in the evaluation of $\mathrm{AD}$, even in the early stages when current techniques still have limited ability to differentiate $\mathrm{AD}$ from other age-related dementias. Even though there are different neuropathology related tests, various imaging modalities, biomarkers and drug therapies etc for the diagnosis of AD, they are insufficient for a definite diagnosis. But if we can combine the features of all of the above using soft computing techniques such as fuzzy logic, neural computing, evolutionary computation and probabilistic reasoning, it may be possible to early diagnosis of the disease in a convenient way by making an expert system. Fuzzy logic can handle exactness, neural networks focus on learning, evolutionary computation deals with the design and operation of a system to make it as good as possible and probabilistic reasoning can handle the condition of being uncertain.

\section{Conclusion}

The newest investigations made on retina of $\mathrm{AD}$ patients provide a breakthrough in the initial screening, early diagnosis or monitoring the treatment of $\mathrm{AD}$ by considering retina as a biomarker. Also a definite diagnosis of $\mathrm{AD}$ can done through autopsy diagnosis of $\mathrm{AD}$ .There are a lot of clinical tests, drug therapies and diagnostic tools such as biomarkers and imaging techniques are available for the diagnosis of Alzheimer's disease. But the fact is that these techniques are inadequate for the definite diagnosis at the earlier stages. So a newly reliable and efficient method should be developed in order to diagnose the disease with the advanced Biomedical Engineering technology using the aid of various clinical tests, imaging techniques such as SPECT, MRI, PET, OCT etc, databases and soft computing tools. With the help of above methods profiling of human body parameters for diagnosing $\mathrm{AD}$ can be made. The screening tests for identifying the $\mathrm{AD}$ patients early can be conducted with minimum effort. A clinical follow up for carry out the diagnosis can be set with the above approaches. The AD conformation test can be made with minimum cost and time. The early prediction of $\mathrm{AD}$ can be made with the above mentioned methods in a reliable and effective way. As we know that this disease is progressing worldwide with no suitable diagnosis, an effective approach towards this can be made with a point of view to diagnose $\mathrm{AD}$ with the minimum effort, cost and time.

\section{Acknowledgments}

None.

\section{Conflicts of Interset}

None.

\section{References}

1. Sandeep CS, Sukesh KumarA, Mahadevan K, et al. Dimensionality Reduction of Optical Coherence Tomography Images for the Early Diagnosis of Alzheimer's Disease. American Journal of Electrical and Electronic Engineering. 2017;5(2): 58-63.

2. Sandeep CS, Sukesh Kumar A. A Review on the Early Diagnosis of
Alzheimer's Disease (AD) through Different Tests, Techniques and Databases. AMSE Journal. 2015;76(1):1-22.

3. Sandeep CS, Sukesh Kumar A, Susanth MJ. The Online Datasets Used to Classify the Different Stages for the Early Diagnosis of Alzheimer's Disease (AD). International Journal of Engineering and Advanced Technology. 2017;6(4):38-45.

4. Sandeep CS, Sukesh Kumar A. A Psychometric Assessment Method for the Early Diagnosis of Alzheimer's disease. International Journal of Scientific \& Engineering Research. 2017;8(3):901-905.

5. Frost S, Martins RN, Kanagasingam Y. Ocular biomarker for early detection of Alzheimer's disease. J Alzheimers Dis. 2010;22(1):1-16.

6. Ohno-Matsu K. Parallel findings in age-related macular degeneration and Alzheimer's disease. Prog Retin Eye Res. 2011;30(4):217-238.

7. Locascio JJ, Growdon JH, Corkin S. Cognitive test performance in detecting, staging, and tracking Alzheimer's disease. Arch Neurol. 1995;52(11):1087-1099.

8. Paque L, Warrington EK. Alongitudinal study of reading ability in patients suffering from dementia. J Int Neuropsychol Soc. 1995;1(6):517-524.

9. Farrer L, Brin M, Elsas L, et al. Statement on the use of apolipoprotein E testing for Alzheimer disease (AD). JAMA. 1995;274(20):1627-1629.

10. Bradshaw JR, Thomson JLG, Campbell MJ. Computed tomography in the investigation of dementia. BMJ. 1983;286(6361):277-280.

11. Katzman R. Should a major imaging procedure (CT or MRI) be required in the workup of dementia?: an affirmative view. J Fam Pract. 1990;31(4):401-410.

12. Ivnik RJ, Malec JF, Smith GE, et al. Neuropsychological tests' norms above age 55: COWAT, BNT, MAE Token, WRAT-R Reading, AMNART, STROOP, TMT, and JLO. Clin Neuropsychol. 1996;10(3):262-278.

13. Becker PM, Feussner JR, Mulrow CD, et al. The role of lumbar puncture in the evaluation of dementia: the Durham Veterans Administration/ Duke University study. J Am Geriatr Soc. 1985;33(6):392-396.

14. Krasodomska K, Lubinski W, Potemkowski A, et al. Pattern electroretinogram (PERG) and pattern visual evoked potential (PVEP) in the early stages of Alzheimer's disease. Doc Ophthalmol. 2010;121(2):111-121.

15. Oliveira LT, Louzada PR, Mello FG, et al. Amyloid-b decreases nitric oxide production in cultured retinal neurons:a possible mechanism for synaptic dysfunction in Alzheimer's disease? Neurochem Res. 2011;36:163-169.

16. Berisha F, Feke GT, Trempe CL, et al. Retinal Abnormalities in early Alzheimer's disease. Invest Ophthalmol Vis Sci. 2007;48(5):2285-2289.

17. Kesler A, Vakhapova V, Korczyn AD, et al. Retinal thickness in patients with mild cognitive impairment and Alzheimer's disease. Clin Neurol Neurosurg. 2011;113(7):523-526.

18. Guo L, Duggan J, Corderio MF. Alzheimer's disease and retinal neurodegeneration. Curr Alzheimer Res. 2010;7(1):3-14.

19. McKee A, Au R, Cabral H, et al. Visual association pathology in preclinical Alzheimer disease. J Neuropathol Exp Neurol. 2006;65(6):621-630.

20. Valenti DA. Anterior Visual System and circadian function with reference to Alzheimer's disease. Vision in Alzheimer's disease. 2006;34:1-29.

21. Shabana N, Cornilleau P, Carkeet A, et al. Motion perception in glaucoma patients: a review. Surv Ophthalmol. 2003;48(1):92-106.

22. Mei M, Leat S. Suprathreshold contrast matching in maculopathy. Invest Ophthalmol Vis Sci. 2007;48(7):3419-3424.

23. Feigl B, Brown B, Lovie-Kitchin J, et al. Monitoring retinal function in early age-related maculopathy: visual performance after one year. Eye (Lond). 2005;19(11):1169-1177. 
24. Cronin-Golomb A, Rizzo J, Corkin S, et al. Visual function in Alzheimer's disease and normal aging. Ann NY Acad Sci. 1961;640:28-35.

25. Thiyagesh SN, Farrow TFD, Parks RW, et al. The neural basis of visuospatial perception in Alzheimer's disease and healthy elderly comparison subjects: an fMRI study. Psychiatry Res. 2009; 172(2):109-116.

26. Mapstone M, Dickerson K, Duffy CJ. Distinct mechanisms of impairment in cognitive ageing and Alzheimer's disease. Brain. 2008;131(6):1618-1629.
27. Gilmore GC, Wend HE, Naylor L, et al. Motion perception and Alzheimer's disease. J Gerontol. 1964;49(2):P52-P57.

28. Armstrong R. Visual field defects in Alzheimer's disease patients may reflect differential pathology in the primary visual cortex. Optom Vis Sci. 1966;73(11):677-682.

29. Trick G, Trick L, Morris P, et al. Visual field loss in senile dementia of the Alzheimer's type. Neurology. 1995;45(1):68-74. 